\title{
Risk factors for post-stroke depression in Sohag University Hospital
}

\author{
Gharib Fawi Mohammed, Hemaid Mostafa Azab, Mohamed Abd-Elmoneim Sayed, Hassan Mohammed Elnady*, \\ Hanan Youssif and Ossama Abd-Alreheem Mahmoud
}

\begin{abstract}
Background: Depression is one of the important complications of stroke. Post-stroke depression is associated with more physical disability, especially in daily activities, poor functional outcome, and high mortality rate.

Objective: Our study aimed to investigate the risk factors for development of post-stroke depression 3 months following stroke.

Methods: This is a comparative study, comparing stroke patients with and without depression 3 months after the onset. Hamilton Depression Rating Scale (HDRS), Mini-Mental State Examination (MMSE), Scandinavian Stroke Scale (SSS), and modified Rankin scale (mRS) were used to evaluate patients 3 months post-stroke for depression, cognition, and physical disability. The patients were divided into two groups: patients' group with depression and patients' group without depression according to DSM-IV criteria. The clinical characteristics and scores on the HDRS, MMSE, SSS, and mRS were compared between depressive group and non-depressive group. Logistic regression analysis was performed to identify risk factors for depression 3 months after stroke.
\end{abstract}

Results: A total 102 stroke patients were recruited. Of these, 62 patients (60.78\%) had depression. Multivariate logistic regression indicated that smoking, hypertension, increased physical disability, and impaired cognitive function were associated with post-stroke depression.

Conclusion: Important risk factors found for PSD included smoking, hypertension, increased physical disability, and impaired cognitive function.

Keywords: Stroke, Depression, Risk factors

\section{Introduction}

Stroke is related to high morbidity and mortality rate throughout the world. Its sudden onset and its remaining mental or physical sequelae have a negative effect on whatever is left of life [1].

Depression is one of the critical complications of stroke [2]. Its prevalence was evaluated to be from 20 to $65 \%$, contingent upon the population studied, the assessment tools, and the defining criteria of depression [3]. Post-stroke depression (PSD) is associated with more physical disability, particularly in day by day activities, poor functional outcome, and high death rate [4].

Previous history of depression, the severity of neurological deficit, functional dependency, and pre-stroke

\footnotetext{
* Correspondence: elnadyhassan1974@hotmail.com; hass_elnady@yahoo.com Department of Neurology and Psychological Medicine, Faculty of Medicine, Sohag University, Sohag, Egypt
}

\section{Material and methods}

This is a comparative study, comparing first ever stroke patients with and without depression 3 months after the onset. The cases came from a series of consecutive patients attending the Neurology Outpatient Clinic of Sohag University Hospital from February 2012 to March 2013. The total sample was divided into two groups, 
stroke patients presented with (group A) and without (group B) depression. Stroke was defined according to the definition of the World Health Organization as the rapid onset of a new persistent neurological deficit attributable to an obstruction in cerebral blood flow with no apparent non-vascular cause [6]. Patients with aphasia, mental illness (e.g., schizophrenia or dementia), substance-related disorders, chronic organic illness (e.g., renal or hepatic failure), or patients using SSRI were excluded. Consents were obtained from participants. The study was approved by the local Ethical Committee of Sohag Faculty of Medicine, Sohag University. Written consent was obtained from all participants.

The following data were collected (from patients' files during admission): age, gender, smoking, hypertension, hypercholesterolemia, diabetes mellitus, history of cardiac disease, including rheumatic heart disease, atrial fibrillation, coronary artery disease, myocardial infarction, heart failure, and positive family history for cerebrovascular stroke. Patients were categorized as hypertensive if their blood pressure readings preceding the index stroke had repeatedly surpassed $140 / 95 \mathrm{mmHg}$, or if they were taking medication to control hypertension, the subjects were considered to be diabetics if they used oral hypoglycemic agents or insulin, and as having heart disease when known before admission or diagnosed on admission. Clinical data on admission were collected, including systolic and diastolic blood pressure, admission temperature, pulse rate and its rhythm, the presence of hiccough or vomiting, side of weakness, the presence of aphasia, among non-comatose patients, severity of stroke using the Glasgow Coma Scale (GCS), and Scandinavian Stroke Scale [7]. The following laboratory data were evaluated: blood sugar, serum creatinine, complete blood count, coagulation profile, erythrocyte sedimentation rate, serum electrolytes, and liver function tests. Radiological data, on admission, were also evaluated to determine the type and site of cerebrovascular stroke.

\section{All cases assessed using}

Hamilton Depression Rating Scale (Arabic version) [8] was performed by a skillful neuropsychologist who carried out the interview to diagnose depression within 3 months after stroke onset according to DSM-IV criteria. The severity of depression was evaluated utilizing the 17-item Hamilton Rating Scale for Depression (HAMD17) within 3 months after stroke onset [9]. To be incorporated into the major depression group in our final analysis, subjects needed to meet DSM-IV criteria for major depressive disorder. The other participants were allotted to either the control group.

Mini-Mental State Examination [10] is a broadly utilized cognitive screening test, where scores from 24 to
30 are considered within the normal range. Items address orientation, memory, recall, attention, naming objects, following verbal and written commands, writing a sentence, and copying a figure.

The outcome was assessed at 3 months after the stroke by clinical examination utilizing the modified Rankin scale (mRS). The mRS scale scores of 0 to 1 were categorized as "favorable" and mRS scores of 2 to 6 as "unfavorable" outcome [11-13].

\section{Statistical methods}

Data was analyzed using STATA intercooled version 9.2. Categorical variables were expressed as frequency and percentage while continuous variables were expressed as mean \pm standard deviation. Quantitative data were analyzed using the Mann-Whitney $U$ test to compare two groups as the data was not normally distributed. Qualitative data were compared using either chi-square test or Fisher exact test. Simple logistic regression analysis was used to identify factors predicting depression. A logistic regression analysis, which included variables that showed statistical differences on univariate comparison, was performed.

\section{Results}

During the study period, 102 patients were eligible for analysis (43 males and 59 females) with age ranging from 18 to 87 years presented 3 months post-stroke. Sixty-two patients were categorized as group A and 40 patients as group B. There was no statistically significant difference between patients with and without depression as regard to age, sex, and type of stroke as shown in (Table 1).

Smoking was more prevalent in group A than in group B $(p=0.03)$. Patients with depression were more often hypertensive $(p$ value $<0.003)$ and diabetic $(p$ value $<$ 0.01 ). There was no statistically significant difference between two groups as regards the prevalence of cardiac disease ( $p$ value 0.10 ), laboratory findings, and side or site of lesion as shown in (Table 2).

Patients in group A were more likely to have impaired cognitive function than those in group $\mathrm{B}$, and the difference was statistically significant ( $p$ value $<0.0001)$. A higher stroke severity, measured by Scandinavian Stroke Scale, was significantly higher in patients with depression $(p$ value $<0.005)$. The prevalence of unfavorable clinical outcome assessed with the mRS was significantly higher in group A ( $p$ value $<0.0001)$ (Table 3$)$.

Table 4 shows the multivariate analysis using logistic regression model and odds ratios for each of the factors. The model indicated that smoking $(p$ value $<0.03$ ), hypertension ( $p$ value $<0.01$ ), increased physical disability (unfavorable outcome) according to mRS ( $p$ value < 0.01 ), higher stroke severity ( $p$ value 0.01 ), and impaired 
Table 1 Demographic data and type of stroke in both groups

\begin{tabular}{|c|c|c|c|c|c|}
\hline & \multicolumn{2}{|c|}{$\begin{array}{l}\text { Group A } \\
\text { Patients with depression }\end{array}$} & \multicolumn{2}{|c|}{$\begin{array}{l}\text { Group B } \\
\text { Patients without depression }\end{array}$} & \multirow[t]{3}{*}{$p$ value } \\
\hline & Number & Percentage & Number & Percentage & \\
\hline & 62 & $60.78 \%$ & 40 & $39.22 \%$ & \\
\hline Age (mean (SD) & \multicolumn{2}{|l|}{$56.67(10.43)$} & \multicolumn{2}{|l|}{$56.7(13.03)$} & 0.30 \\
\hline \multicolumn{6}{|l|}{ Sex } \\
\hline Females & 35 & $59.32 \%$ & 24 & $40.06 \%$ & \multirow[t]{2}{*}{0.69} \\
\hline Males & 27 & $62.79 \%$ & 16 & $37.20 \%$ & \\
\hline \multicolumn{6}{|l|}{ Type of stroke } \\
\hline Hemorrhage & $n=13$ & $68.42 \%$ & $n=6$ & $31.57 \%$ & \multirow[t]{2}{*}{0.40} \\
\hline Infarction & $n=49$ & $59.30 \%$ & $n=34$ & $40.96 \%$ & \\
\hline
\end{tabular}

cognitive function $(p$ value $<0.01)$ were associated with post-stroke depression.

\section{Discussion}

The interaction between stroke and depression is exceptionally intricate, and the pathophysiological mechanisms have not as yet been fully explained [14].
Various studies explored prevalence and risk factors for PSD, but inconstant results were reported relying on the studied subjects, assessment tools, and the diagnostic criteria of depression used [15].

The prevalence of PSD in our study was 60.78\%, which was higher than previously reported studies [16-18], and in line with other studies, which reported that the prevalence of PSD ranged from 5 to

Table 2 Clinical, laboratory, and radiological data in both groups

\begin{tabular}{|c|c|c|c|c|c|}
\hline & \multicolumn{2}{|c|}{$\begin{array}{l}\text { Group A } \\
\text { Patients with depression }\end{array}$} & \multicolumn{2}{|c|}{$\begin{array}{l}\text { Group B } \\
\text { Patients without depression }\end{array}$} & \multirow[t]{2}{*}{$\overline{p \text { value }}$} \\
\hline & Total $n=62$ & Percentage (\%) & Total $n=40$ & Percentage (\%) & \\
\hline Smoking & 37 & 75.75 & 8 & 24.24 & $<0.03$ \\
\hline Hypertension & 32 & 78.04 & 9 & 21.95 & $<0.003$ \\
\hline Diabetes mellitus & 36 & 78.78 & 7 & 21.21 & $<0.01$ \\
\hline Cardiac disease & 9 & 47.36 & 10 & 52.63 & 0.10 \\
\hline Hypercholesterolemia & 19 & 59.37 & 13 & 40.62 & 0.12 \\
\hline Hypertriglyceridemia & 14 & 60.87 & 9 & 39.13 & 0.15 \\
\hline Low HDLC & 41 & 60.29 & 27 & 39.70 & 0.15 \\
\hline High LDLC & 12 & 60.00 & 8 & 40.00 & 0.15 \\
\hline Side of the lesion & & & & & 0.45 \\
\hline Free $\mathrm{CT}$ brain on admission & $n=8$ & 53.33 & $n=7$ & 46.66 & \\
\hline Left cerebral hemisphere & $n=12$ & 40.00 & $n=18$ & 60.00 & \\
\hline Right cerebral hemisphere & $n=20$ & 35.08 & $n=37$ & 64.91 & \\
\hline \multicolumn{6}{|l|}{ Site of lesion } \\
\hline Free $C T$ brain on admission & 8 & 53.33 & 7 & 46.66 & 0.52 \\
\hline Basal ganglionic & 8 & 34.74 & 15 & 65.21 & \\
\hline Cerebellar & 2 & 50.00 & 2 & 50.00 & \\
\hline Thalamic & 1 & 25.00 & 3 & 75.00 & \\
\hline Frontal & 7 & 38.88 & 11 & 61.11 & \\
\hline Occipital & 1 & 33.33 & 2 & 66.66 & \\
\hline Parietal & 13 & 46.42 & 15 & 53.57 & \\
\hline Temporal & 3 & 42.85 & 4 & 57.14 & \\
\hline
\end{tabular}


Table 3 Different applied scales in both groups

\begin{tabular}{llll}
\hline & $\begin{array}{l}\text { Group A } \\
\text { Patients with depression } \\
\text { total } n=62\end{array}$ & $\begin{array}{l}\text { Group B } \\
\text { Patients without depression total } n=40\end{array}$ & $p$ value \\
\hline MMS: mean (SD) & $26.93(2.74)$ & $24.89(2.09)$ & $<0.0001$ \\
SSS score: mean (SD) & $39.8(13.71)$ & $30.63(11.09)$ & 0.005 \\
mRS: mean (SD) & $2.05(1.22)$ & $3.00(0.75)$ & $<0.0001$ \\
\hline
\end{tabular}

63\% [19] and from 25 to $79 \%$ [20]. Differences in reported prevalences of previous studies are likely a result of differences in methods of measurement and a function of the timing of the evaluation [13].

Because of the unfavorable impacts of PSD, it is essential to distinguish risk factors to allow for an early diagnosis and management, in this way diminishing the negative impacts in stroke patients [21].

In the current study, there was no relationship between the demographic data (age and sex) and PSD. The results for the relationship between PSD and demographic data are controversial [22]. Some of the earlier reports are in agreement with our results [5, 23], while others are not $[21,24]$.

Contrary to our results, it was reported that patients under 50 years old occupy a higher percentage of subjects with depression [23]. Also, it was recognized that younger patients appear to be more attentive about the prognosis of the disease $[4,25]$.

In an Egyptian study, the authors found that male gender, younger age, and frontal lesions were predictors for post-stroke depression [26].

In another Egyptian study, to detect the depression, anxiety, and personality traits in post-stroke patient, there was no significant relation between demographic data and depression in post-stroke patients [27].

Although smoking and hypertension were established risk factors for development of stroke [28], they were not reported as risk factors for development of PSD [13, 29].

Among our subjects, smoking was related to PSD in univariate and multivariate analysis. This is in accord with a study by Ren and colleagues who reported that the frequency of depression in the smoker group was

Table 4 Logistic regression model of significant risk factors associated with post-stroke depression

\begin{tabular}{lll}
\hline & \multicolumn{2}{l}{ Multivariate analysis } \\
\cline { 2 - 3 } & Odds ratio (95\% Cl) & $p$ value \\
\hline Smoking & $3.56(1.10-11.52)$ & 0.03 \\
Hypertension & $4.76(1.42-15.92)$ & 0.01 \\
Modified Rankin scale score & $3.72(1.30-10.69)$ & 0.01 \\
Scandinavian Stroke Scale score & $4.84(1.40-16.70)$ & 0.01 \\
Mini-Mental State of Examination & $3.75(1.18-11.90)$ & 0.01 \\
\hline
\end{tabular}

significantly higher than that in the non-smoker group [30]. In addition, Ren and colleagues reported that, the larger proportion of patients with depression in smokers with acute ischemic stroke may be related to lower vitamin D levels induced by smoking [30].

In our study, patients with post-stroke depression had a significant functional disability in (cognition, physical activity) compared to stroke patients without depression, which was consistent with many previous studies that had reported a significant relationship between disability and post-stroke depression [15, 31-34].

De Ryck and colleagues [13] conducted a prospective, longitudinal epidemiological study in 135 patients who completed follow-up assessments at 3 months post-stroke; they concluded that the most determining features for depression risk after stroke included reduced mobility and cognitive impairment. Leentjens and colleagues [35] assessed 190 consecutively admitted patients for major depressive disorder 1 month after stroke, and at follow-up after 3, 6, 9, and 12 months, they revealed that disability was associated with PSD. Nys and colleagues [36] studied 143 cases within the first 3 weeks post-stroke; they revealed that cognitive impairment and vascular risk factors were significant predictors of long-term depressive symptoms and quality of life after stroke.

Our study had several limitations. The sample size was small which generates imprecision in some estimates. The study population included both ischemic and hemorrhagic strokes. We did not differentiate types of strokes according to TOAST criteria. In addition, we excluded patients with aphasia because this might limit the generalization of our findings. Moreover, the socioeconomic factors and education were not included in the analysis.

\section{Conclusion}

Depression was found to be an important complication among stroke survivors in our study. Important risk factors found for PSD included smoking, hypertension, increased physical disability, and impaired cognitive function. Early diagnosis and adequate therapy are still necessary in stroke rehabilitation to attenuate stroke complication. 


\section{Abbreviations}

GCS: Glasgow Coma Scale; HDRS: Hamilton Depression Rating Scale; MMSE: MiniMental State Examination; mRS: Modified Rankin scale; PSD: Post-stroke depression; SSRI: Selective serotonin reuptake inhibitors; SSS: Scandinavian Stroke Scale

\section{Acknowledgements \\ Not applicable.}

\section{Funding}

None.

\section{Availability of data and materials}

The data set of this work is available and uploaded with this article.

\section{Authors' contributions}

GF participated in the design of the study and supervised the findings of this work. HA conceived of the study and participated in its design and coordination. MS analyzed the data and was involved in the study design. $\mathrm{HE}$ and HY wrote the paper, drafted the manuscript, and designed the tables. OA was involved in the data acquisition and analysis. All authors discussed the results and commented on the manuscript. All authors read and approved the final manuscript.

\section{Ethics approval and consent to participate}

The study was approved by the local Ethical Committee of Sohag Faculty of Medicine, Sohag University on December 13, 2011, Informed written (when available) consent was obtained from all patients for participation and publication of this study.

\section{Consent for publication}

Not applicable.

\section{Competing interests}

The authors declare that they have no competing interests.

\section{Publisher's Note}

Springer Nature remains neutral with regard to jurisdictional claims in published maps and institutional affiliations.

Received: 12 November 2017 Accepted: 7 January 2019

\section{Published online: 28 January 2019}

\section{References}

1. Li CT, et al. Major depressive disorder and stroke risks: a 9-year follow-up population-based, matched cohort study. PLoS One. 2012;7(10):e46818.

2. Hackett ML, Anderson CS. Predictors of depression after stroke: a systematic review of observational studies. Stroke. 2005;36(10):2296-301.

3. De Ryck A, et al. Risk factors for poststroke depression: identification of inconsistencies based on a systematic review. J Geriatr Psychiatry Neurol. 2014;27(3):147-58.

4. Karamchandani RR, et al. Early depression screening is feasible in hospitalized stroke patients. PLoS One. 2015;10(6):e0128246.

5. dos Santos EB, Rodrigues RAP, Pontes-Neto OM. Prevalence and predictors of post stroke depression among elderly stroke survivors. Arq Neuropsiquiatr. 2016;74(8):621-5.

6. Hatano S. Experience from a multicentre stroke register: a preliminary report. Bull World Health Organ. 1976;54(5):541-53.

7. Gray LJ, et al. Interconversion of the National Institutes of Health Stroke Scale and Scandinavian Stroke Scale in acute stroke. J Stroke Cerebrovasc Dis. 2009;18(6):466-8.

8. Lotfy H. Hamilton Rating Scale of depression (HDRS). Cairo: Dar El-Anglo; 1994.

9. Hamilton M. Development of a rating scale for primary depressive illness. $\mathrm{Br}$ J Soc Clin Psychol. 1967;6(4):278-96.

10. Multicenter trial of hemodilution in ischemic stroke--background and study protocol. Scandinavian Stroke Study Group. Stroke. 1985;16(5):885-90.

11. Bonita $R$, Beaglehole R. Recovery of motor function after stroke. Stroke. 1988;19(12):1497-500.

12. van Swieten JC, et al. Interobserver agreement for the assessment of handicap in stroke patients. Stroke. 1988;19(5):604-7.
13. De Ryck A, et al. A prospective study on the prevalence and risk factors of poststroke depression. Cerebrovasc Dis Extra. 2013;3(1):1-13.

14. Zhang $Y$, et al. Neural complexity in patients with poststroke depression: a resting EEG study. J Affect Disord. 2015;188:310-8.

15. Oliveira $C R$, et al. Depressive signs and cognitive performance in patients with a right hemisphere stroke. Codas. 2015;27(5):452-7.

16. Kouwenhoven SE, et al. Depressive symptoms in acute stroke: a crosssectional study of their association with sociodemographics and clinical factors. Neuropsychol Rehabil. 2013;23(5):658-77.

17. Snaphaan $L$, et al. Post-stroke depressive symptoms are associated with post-stroke characteristics. Cerebrovasc Dis. 2009:28(6):551-7.

18. Johnson $\mathrm{J}$, et al. Poststroke depression incidence and risk factors: an integrative literature review. J Neurosci Nurs. 2006;38(4 Suppl):316-27.

19. Hadidi N, Treat-Jacobson DJ, Lindquist R. Poststroke depression and functional outcome: a critical review of literature. Heart Lung. 2009;38(2): 151-62.

20. Hosking SG, Marsh NV, Friedman PJ. Depression at 3 months poststroke in the elderly: predictors and indicators of prevalence. Aging Neuropsycho Cogn. 2000;7(4):205-16.

21. Tsai CS, et al. Incidence and risk factors of poststroke depression in patients with acute ischemic stroke: a 1-year prospective study in Taiwan. Biom J. 2016:39(3):195-200.

22. Kutlubaev MA, Hackett ML. Part II: predictors of depression after stroke and impact of depression on stroke outcome: an updated systematic review of observational studies. Int J Stroke. 2014;9(8):1026-36.

23. Jiao JT, et al. Association between inflammatory cytokines and the risk of post-stroke depression, and the effect of depression on outcomes of patients with ischemic stroke in a 2-year prospective study. Exp Ther Med. 2016;12(3):1591-8

24. Tang WK, et al. Poststroke depression in Chinese patients: frequency, psychosocial, clinical, and radiological determinants. J Geriatr Psychiatry Neurol. 2005;18(1):45-51.

25. Carota A, et al. A prospective study of predictors of poststroke depression. Neurology. 2005;64(3):428-33.

26. Effat SM, Mohamed MM, El Essawy HI, El Sheikh MM, Abdul Aal HS. Predictors and Consequences of Post-Stroke Depression in a Sample of Egyptian. Arab J Psychiatry. 2011;22(1):19-26.

27. Shehata GA, Hassan HS. Personality traits, depression and anxiety among cerebro-vascular stroke patients: an Egyptian study. Int Neuropsychiatric Dis J. 2014;2(6):280-8

28. Park $\mathrm{TH}$, et al. Identifying target risk factors using population attributable risks of ischemic stroke by age and sex. J Stroke. 2015;17(3):302-11.

29. Yang SR, et al. Predictors of early post ischemic stroke apathy and depression: a cross-sectional study. BMC Psychiatry. 2013;13:164

30. Ren $W$, et al. The effect of cigarette smoking on vitamin D level and depression in male patients with acute ischemic stroke. Compr Psychiatry. 2016:65:9-14

31. Srivastava A, et al. Post-stroke depression: prevalence and relationship with disability in chronic stroke survivors. Ann Indian Acad Neurol. 2010;13(2): 123-7.

32. Paolucci $S$, et al. The Italian multicenter observational study on post-stroke depression (DESTRO). J Neurol. 2006;253(5):556-62.

33. Glodzik-Sobanska $L$, et al. Depressive symptoms following ischemic stroke. Neurol Neurochir Pol. 2003;37(1):17-25.

34. Parikh RM, et al. Two-year longitudinal study of post-stroke mood disorders: dynamic changes in correlates of depression at one and two years. Stroke. 1987;18(3):579-84

35. Leentjens AF, et al. General and disease-specific risk factors for depression after ischemic stroke: a two-step Cox regression analysis. Int Psychogeriatr. 2006;18(4):739-48.

36. Nys GM, et al. Early cognitive impairment predicts long-term depressive symptoms and quality of life after stroke. J Neurol Sci. 2006;247(2):149-56. 